\title{
Glutamate Receptors in Perirhinal Cortex Mediate Encoding, Retrieval, and Consolidation of Object Recognition Memory
}

\author{
Boyer D. Winters and Timothy J. Bussey \\ Department of Experimental Psychology, University of Cambridge, Cambridge CB2 3EB, United Kingdom
}

\begin{abstract}
Object recognition is consistently impaired in human amnesia and animal models thereof. Results from subjects with permanent brain damage have revealed the importance of the perirhinal cortex to object recognition memory. Here, we report evidence from rats for interdependent but distinct stages in object recognition memory (encoding, retrieval, and consolidation), which require glutamate receptor activity within perirhinal cortex. Transient blockade of AMPA receptor-mediated synaptic transmission within perirhinal cortex disrupted encoding for short- and long-term memory as well as retrieval and consolidation. In contrast, transient NMDA receptor blockade during encoding affected only long-term object recognition memory; NMDA receptor activity was also necessary for consolidation but not retrieval. These results further demonstrate the importance of perirhinal cortex for object recognition memory and suggest that, as in the hippocampus, AMPA and NMDA receptors mediate synaptic transmission and activity-dependent synaptic plasticity, respectively, in several stages of memory processing.
\end{abstract}

Key words: acquisition; AMPA; declarative memory; NMDA; rat; storage

\section{Introduction}

Visual recognition paradigms have played an indispensable role in our understanding of the neurobiological basis of memory (Mishkin, 1978; Zola-Morgan and Squire, 1985; Zola-Morgan et al., 1986; McKee and Squire, 1993; Bartolini et al., 1996; Ennaceur et al., 1996; Buffalo et al., 1998; Elliott and Dolan, 1998; Murray and Mishkin, 1998; Pascalis and Bachevalier, 1999; Tang et al., 1999; Bozon et al., 2003; Duzel et al., 2003; Nemanic et al., 2004). There is now strong evidence that the perirhinal cortex $(\mathrm{PRh})$ is a critical structure required for the performance of such tasks (Zola-Morgan et al., 1989; Meunier et al., 1993; Gaffan, 1994; Mumby and Pinel, 1994; Buffalo et al., 1998; Bussey et al., 1999, 2000; Murray and Bussey, 1999; Winters et al., 2004). Much of what is known, however, about the role of PRh in object recognition memory is based on findings from subjects with permanent PRh damage. Although valuable, such reports cannot unambiguously address the issue of the role of PRh in distinct memory stages such as encoding, retrieval, and consolidation. In contrast, transient pharmacological manipulations within PRh could elucidate more specific functions of this temporal lobe region and the transmitter systems that mediate its involvement in object recognition memory. Indeed, we recently demonstrated involvement of $\mathrm{PRh}$ in encoding, retrieval, and consolidation of object recognition memory using transient lidocaine-induced in-

\section{Received Nov. 18, 2004; revised March 17, 2005; accepted March 20, 2005}

This work was supported by a Biotechnology and Biological Sciences Research Council research grant to T.J.B., B.D.W., and Dr. Lisa M. Saksida and a Wellcome Trust Project grant to T.J.B. and Dr. Saksida. This research was conducted under the auspices of the Cambridge Medical Research Council Centre for Behavioral and Clinical Neuroscience.

Correspondence should be addressed to Dr. B. D. Winters, Department of Experimental Psychology, University of Cambridge, Downing Street, Cambridge CB2 3EB, UK. E-mail: bdw23@cam.ac.uk.

DOI:10.1523/JNEUROSCI.0480-05.2005

Copyright $\odot 2005$ Society for Neuroscience $\quad$ 0270-6474/05/254243-09\$15.00/0 activation at different stages of the spontaneous object recognition task for rats (Winters and Bussey, 2005).

The glutamatergic system is important for fast synaptic transmission, via AMPA receptors (AMPARs), and activitydependent synaptic plasticity, which may underlie long-term memory (Bliss and Lomo, 1973; Martin et al., 2000). The induction of certain activity-dependent synaptic plasticity processes depends crucially on NMDA glutamate receptors (NMDARs) (Bliss and Collingridge, 1993). The different functions of AMPARs and NMDARs prompt unique predictions about their respective roles in the stages of memory processing. Indeed, a recent study revealed the importance of AMPAR-mediated hippocampal synaptic transmission in encoding, retrieval, and consolidation of spatial memory (Riedel et al., 1999). Another study has shown that blockade of hippocampal NMDARs disrupts encoding, but not retrieval, in a paired-associates task, whereas AMPAR blockade interferes with both processes (Day et al., 2003). These findings are consistent with roles for AMPARs and NMDARs in synaptic transmission and induction of activitydependent synaptic plasticity, respectively.

The goal of the present study was to examine further the role of PRh in the distinct stages of object recognition memory by assessing the possible differential involvement of AMPARs and NMDARs within PRh in encoding, retrieval, and consolidation. We have capitalized on the discrete one-trial nature of the spontaneous object recognition paradigm (Ennaceur and Delacour, 1988) to test the effects of transient blockade of AMPARs or NMDARs during sample presentation (encoding), during the choice phase (retrieval), and during the retention interval (consolidation). We report dissociable functions for these two glutamate receptor types in PRh-mediated object recognition memory and an important role for both AMPARs and NMDARs in memory consolidation within PRh. 


\section{Materials and Methods}

\section{Subjects}

The subjects were 46 adult male Lister hooded rats (Harlan Olac, Bicester, UK), weighing 270-320 g before surgery and housed in pairs in a room with a $12 \mathrm{~h}$ light/dark cycle (lights on at 7:00 P.M.). Different batches of rats were used for each experiment. The number of rats used in each experiment was as follows: experiment 1, 11 rats; experiment 2, 11 rats; experiment 3,12 rats; experiment 4, 12 rats. All behavioral testing was conducted during the dark phase of the cycle. During testing, rats were fed $\sim 15$ g of laboratory chow after daily behavioral sessions to maintain weights at $85-90 \%$ of free-feeding body weight. Water was available ad libitum throughout the experiment. All experimentation was conducted in accordance with the United Kingdom Animals (Scientific Procedures) Act (1986).

\section{Surgery}

For each experiment, all rats were implanted bilaterally with 22-gauge indwelling guide cannulas according to the following procedure. Before surgery, all animals were anesthetized deeply by intraperitoneal injection $(60 \mathrm{mg} / \mathrm{kg}$, i.p.) of sodium pentobarbital (Sagatal; Rhône Mérieux, Essex, UK) and placed in a stereotaxic frame (David Kopf Instruments, Tujunga, CA) with the incisor bar set at $-3.2 \mathrm{~mm}$. The scalp was cut and retracted to expose the skull. Holes were drilled and the guide cannulas implanted according to the following coordinates, measured relative to the skull at bregma (Paxinos and Watson, 1997): anteroposterior, -5.5 $\mathrm{mm}$; lateral, $\pm 6.6 \mathrm{~mm}$; dorsoventral, $-6.5 \mathrm{~mm}$. The cannulas were secured to the skull using four jeweler screws and dental acrylic. Obdurators cut to extend $1.1 \mathrm{~mm}$ beyond the tip of the guide cannulas and with an outer diameter of $0.36 \mathrm{~mm}$ were inserted into the guides and remained there except during infusions. At the completion of each surgery, the skin was sutured, and an antibiotic powder (Acramide; Dales Pharmaceuticals, Skipton, UK) was applied. Animals were allowed to recover for at least $7 \mathrm{~d}$ before the beginning of behavioral testing.

\section{Histology}

After behavioral testing, rats were anesthetized by intraperitoneal injection of $2 \mathrm{ml}$ of Euthatal (Rhône Mérieux) and perfused transcardially with $100 \mathrm{ml}$ of PBS, pH 7.4, followed by $250 \mathrm{ml}$ of $4 \%$ paraformaldehyde (PFA), $\mathrm{pH}$ 7.4. The brains were removed, postfixed in $4 \% \mathrm{PFA}$ at $4^{\circ} \mathrm{C}$ for $24 \mathrm{~h}$, and then immersed in $25 \%$ sucrose in PBS until they sank. Coronal sections $(60 \mu \mathrm{m})$ were cut on a freezing microtome through the extent of $\mathrm{PRh}$, and every fifth section was mounted on a gelatin-coated glass slide and stained with cresyl violet. Slides were examined under a light microscope to verify the cannula placements.

\section{Infusion procedure}

For all experiments, rats received bilateral infusions of either physiological saline $(0.9 \%$ sodium chloride, $\mathrm{pH} 7.0$; Aquapharm; Animalcare Limited, York, UK) or drug (see below) on a given trial. For all conditions, the basic procedure was as follows. All infusions took place in a preparation room separate from the behavioral testing area. Animals were restrained gently by the experimenter throughout the infusion process. The obdurators were removed, and the 28 -gauge infusion cannulas, which were cut to extend $1 \mathrm{~mm}$ beyond the tip of the guides, were inserted into the guides. Bilateral infusions were conducted simultaneously using two $1 \mu \mathrm{l}$ Hamilton syringes, which were connected to the infusion cannulas by propylene tubing. The syringes were driven by a Harvard Apparatus (Holliston, MA) precision syringe pump, which delivered $1 \mu \mathrm{l}$ to each hemisphere over $2 \mathrm{~min}$. The infusion cannulas were left in place for an addition $1.5 \mathrm{~min}$ to allow for diffusion of the infusate. The infusion cannulas were then removed, and the obdurators were replaced before the next phase of the procedure (see below for specific experiment parameters). For all experiments, in each of the two habituation sessions before the beginning of behavioral testing, rats experienced a "mock" infusion identical in all aspects to the procedure described above, except that the injection cannulas contained no liquid. This was done to habituate the animals to the general aspects of the infusion procedure, including insertion of the infusion cannulas and the sound of the pump.

\section{Spontaneous object recognition}

Spontaneous object recognition was conducted in a Y-shaped apparatus, as described previously (Winters et al., 2004; Forwood et al., 2005). Briefly, the Y-shaped apparatus had high, homogeneous white walls constructed from Foamalux (Brett Martin, Lancashire, UK) to prevent the rat from looking out into the room and, thereby, maximize attention to the object stimuli. The apparatus was raised $30 \mathrm{~cm}$ from the floor with walls $40 \mathrm{~cm}$ high. Each arm was $27 \mathrm{~cm}$ in length and $10 \mathrm{~cm}$ wide. The start arm contained a guillotine door $18 \mathrm{~cm}$ from the rear of the arm. This provided a start box area within which the rat could be confined at the start of the sample and choice phases of a given trial. The floor and walls of the apparatus were wiped down with a dry paper towel between rats but otherwise were not cleaned during the experiment. A video camera was mounted above the apparatus to record all trials. Triplicate copies were obtained of the objects, which were made of glass, plastic, or metal. For any given trial, the objects in a pair were composed of the same material so that they could not readily be distinguished by olfactory cues. The height of the objects ranged from 5-20 cm, and all objects were affixed to the floor of the apparatus with Blu Tack (Bostik, Stafford, UK) to prevent them from being displaced during a trial. As far as could be determined, the objects had no natural significance for the rats, and they had never been associated with a reinforcer.

General procedure. All rats were habituated in two consecutive daily sessions in which they were allowed to explore the empty Y-shaped apparatus for $5 \mathrm{~min}$. Before being placed in the apparatus, rats experienced a mock infusion procedure, as described above. After the mock infusion, the rats were taken from the preparation area to the testing room and placed in the start box; the guillotine door was then opened to allow the rat to explore the main area of the apparatus. When the rat exited the start box, the guillotine door was lowered to prevent reentry into this area of the apparatus. The experimenter did not begin timing the session until after the rat exited the start box. Testing began $24 \mathrm{~h}$ after the second habituation session. Rats were given a series of test trials (one per day), with a minimum interval of $24 \mathrm{~h}$ between trials. A different object pair was used for each trial for a given animal, and the order of exposure to object pairs as well as the designated sample and novel objects for each pair were counterbalanced within and across groups. The time spent exploring objects was assessed from video recordings of the sample and choice phases. Data were collected by scoring exploratory bouts using a personal computer running a program written in QuickBASIC 4.5. All trials were run with the experimenter blind to the drug treatment conditions.

Object recognition test. Each trial consisted of two phases. In the sample phase, two identical objects (A1 and A2) were placed in the Y-shaped apparatus, one at the end of each exploration arm. The rat was placed in the start box with the guillotine door lowered. The guillotine door was then raised to allow the rat into the exploration area of the maze. When the rat exited the start box, the guillotine door was lowered to prevent reentry, and the sample phase began. The time spent exploring the two objects was scored by an experimenter viewing the rat on a video screen. The cumulative duration of exploratory bouts, the beginning and end of which were indicated by pressing a given key on the computer keyboard, was calculated by the computer program. Exploration of an object was defined as directing the nose to the object at a distance of $<2 \mathrm{~cm}$ and/or touching it with the nose. Turning around or sitting on the object were not considered exploratory behavior. The sample phase ended when the rat had explored the identical objects for a total of $25 \mathrm{~s}$.

At the end of the sample phase, the rat was removed from the Y-shaped apparatus for the duration of the retention delay. After the delay, the rat was placed back in the start box of the Y-shaped apparatus and released into the exploration area for the choice phase. The Y-shaped apparatus now contained an identical copy of the sample (familiar) object (A3) in one arm and a new object (B) in the other. The exploration arms in which the choice objects were placed were counterbalanced between rats and across trials. The rat was allowed to explore the objects for $2 \mathrm{~min}$, at the end of which it was removed and returned to its home cage. The time spent exploring the novel and familiar objects was recorded for the $2 \mathrm{~min}$ of the choice phase, but attention was focused on the first minute, during 
which object discrimination is typically greatest. We calculated a discrimination ratio, the proportion of total exploration time spent exploring the novel object (i.e., the difference in time spent exploring the novel and familiar objects divided by the total time spent exploring the objects), for the first minute of the choice phase on each object recognition trial. This measure takes into account individual differences in the total amount of exploration time.

Experiment 1. Experiment 1 assessed the effects of pre-sample intra$\mathrm{PRh}$ infusions of the NMDA receptor antagonist $\mathrm{D}(-)$-2-amino-5phosphonopentanoic acid (AP-5; $30 \mathrm{~mm}$ in physiological saline; Sigma, Poole, UK) with a short (5 $\mathrm{min})$ and a long $(180 \mathrm{~min})$ retention delay between sample and choice phases. Eleven rats received infusions, as described above, before the sample phase. Animals were placed into the Y-shaped apparatus $\sim 15 \mathrm{~min}$ after the end of the infusion (disengagement of the pump). After sampling the objects for $25 \mathrm{~s}$, each rat was transferred to an individual holding cage in a room adjacent to the testing room for the retention delay. All rats were run for a total of eight trials, receiving saline infusions on one-half of the trials and lidocaine infusions on the other half. One-half of the trials under each drug condition were run with a 5 min retention delay, and the other half involved a $180 \mathrm{~min}$ delay. Both the drug and delay orders were counterbalanced between rats.

Experiment 2. Experiment 2 was identical to experiment 1 in every aspect, except that the AMPA receptor antagonist 6-cyano-7nitroquinoxaline (CNQX; 3 mm in physiological saline; Sigma) was administered instead of AP-5 on drug infusion trials. Eleven rats served as subjects.

Experiment 3. Experiment 3 assessed the effects of pre-choice intraPRh infusions of AP-5 and CNQX with a 180 min retention delay. Twelve rats received infusions $15 \mathrm{~min}$ before the end of the retention delay on six trials each. Each rat was run on two trials with saline, two with AP-5, and two with CNQX. Drug administration orders were counterbalanced between rats.

Experiment 4. Experiment 4 examined the effects of immediate and 40 min post-sample infusions of AP- 5 and CNQX with a 180 min retention delay. Twelve rats received infusions immediately or $40 \mathrm{~min}$ after the sample phase on counterbalanced trials. Each rat was run for 12 trials, half with immediate postsample infusions and the other half with $40 \mathrm{~min}$ postsample infusions. Each rat was run for two trials in each infusion condition with each of the three drug conditions (saline, AP-5, CNQX). Both the drug and infusion condition orders were counterbalanced between rats.

\section{Data analysis}

Group means of three measures taken from object recognition testing (duration of the sample phase, total exploration time in the choice phase, and the discrimination ratio) were analyzed for each experiment. Means from these measures were submitted to two-way ANOVA for experiments 1 (drug by delay), 2 (drug by delay), and 4 (drug by infusion time) and a univariate ANOVA for experiment 3. Paired-samples $t$ tests were used for post hoc analyses in all experiments. All statistical analyses were conducted with a significance level of $\alpha=0.05$.

\section{Results}

\section{Cannula placements}

Rats in all experiments had cannulas implanted bilaterally within PRh to allow delivery of drugs. All rats included in the behavioral analyses had guide cannulas located bilaterally with injection needle tips terminating in PRh near the border between areas 35 and 36 within cortical layers 2-5 (Burwell, 2001). These placements were consistently located between 5.80 and $6.30 \mathrm{~mm}$ posterior to bregma (Fig. 1), the approximate midsection of the rostral-caudal extent of PRh. Figure 2 shows typical cannula tracks in a representative section stained with cresyl violet. The minor mechanical damage seen around the tip of the infusion cannula track was observed in the brains of rats from all experiments, presumably as a result of repeated infusions. There was, however, no indication of any substantial behavioral impact of this minor damage, because performance in later trials did not differ system-



Figure 1. Cannulation of rat PRh. Schematic representation of the infusion needle tip placements from a typical group of animals (experiment $2 ; n=11$ ). These placements are representative of needle tip locations in all animals included in the behavioral analyses of the present study. Cannulas were consistently located between 5.80 and $6.30 \mathrm{~mm}$ posterior to bregma. Some needle tips overlap in the figure.

atically from earlier trials. Moreover, the within-subjects order of saline and drug infusions was always counterbalanced to control for such potential confounds.

\section{Experiment 1: NMDAR blockade during encoding}

We infused saline or the NMDAR antagonist AP-5 into PRh 15 min before the sample phase to assess the effects of NMDAR blockade on object recognition memory with short (5 min) and long $(180 \mathrm{~min})$ retention delays. The total time required to meet the sample object exploration criterion (25 s cumulative) was analyzed, because a group difference at this stage of the trial might influence subsequent recognition performance. This analysis revealed no significant effects of drug $\left(F_{(1,10)}=2.02 ; p>0.10\right)$, delay $\left(F_{(1,10)}=2.12 ; p>0.10\right)$, or the drug by delay interaction $(F<1)$. The means \pm SEM for separate conditions were as follows: saline, $5 \mathrm{~min}=125.06 \pm 20.20 \mathrm{~s}$; saline, $180 \mathrm{~min}=$ $108.81 \pm 13.86 \mathrm{~s} ; \mathrm{AP}-5,5 \mathrm{~min}=145.57 \pm 13.59 \mathrm{~s} ; \mathrm{AP}-5,180$ $\min =130.49 \pm 16.28 \mathrm{~s}$. Furthermore, there was no effect of drug or delay (both $F<1$ ) on the total amount of time spent by rats exploring the objects in the choice phase, nor was the drug by delay interaction significant $\left(F_{(1,10)}=1.12 ; p>0.10\right)$ (means \pm SEM: saline, $5 \mathrm{~min}=14.15 \pm 1.69 \mathrm{~s}$; saline, $180 \mathrm{~min}=14.37 \pm$ $1.13 \mathrm{~s}$; $\mathrm{AP}-5,5 \mathrm{~min}=16.55 \pm 1.79 \mathrm{~s}$; $\mathrm{AP}-5,180 \mathrm{~min}=13.53 \pm$ $1.78 \mathrm{~s})$. In contrast, intra-PRh AP-5 infusions significantly impaired object recognition memory when tested with the long retention interval (Fig. 3). There were significant effects of drug $\left(F_{(1,10)}=16.19 ; p<0.01\right)$ and delay $\left(F_{(1,10)}=14.08 ; p<0.01\right)$ and a significant drug by delay interaction $\left(F_{(1,10)}=13.54 ; p<\right.$ $0.01)$. Post hoc tests confirmed that animals were significantly 




Figure 2. Photomicrograph illustrating typical cannula tracks in PRh of a representative brain section ( $\sim 5.5 \mathrm{~mm}$ posterior to bregma). The tracks of the guide cannula and the infusion cannula tip are clearly visible.



Figure 3. NMDAR blockade in PRh impairs long- but not short-term object recognition memory. Spontaneous object recognition performance by animals in experiment 1 on trials in which they received intra-PRh infusions of AP-5 or saline 15 min before the sample phase. Rats were impaired on trials with AP-5 infusions when the retention delay between the sample and choice phases was $180 \mathrm{~min}$ but not when the delay was $5 \mathrm{~min}$. Data are presented as average discrimination ratio \pm SEM. ${ }^{* * *} p<0.001, \mathrm{AP}-5$ versus saline. The dashed line indicates chance performance (i.e., zero discrimination between familiar and novel objects).

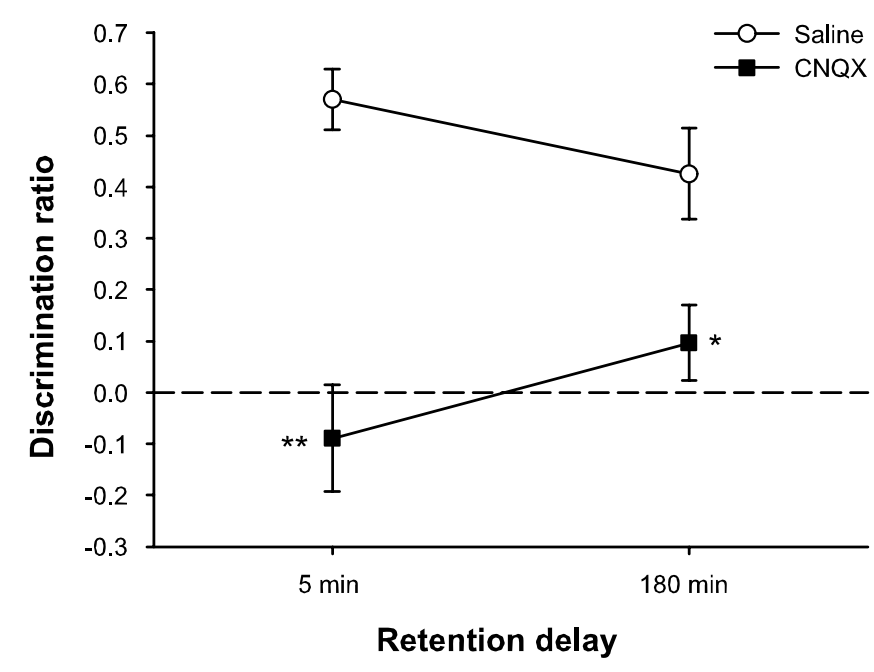

Figure 4. AMPAR blockade in PRh disrupts short-and long-term object recognition memory. Spontaneous object recognition performance by animals in experiment 2 on trials in which they received intra-PRh infusions of CNQX or saline 15 min before the sample phase. Rats were impaired on trials with CNQX infusions regardless of the length of the retention delay. Data are presented as average discrimination ratio $\pm S E M .{ }^{*} p<0.05$, CNQX versus saline; ${ }^{* *} p<0.01$, CNQX versus saline. The dashed line indicates chance performance (i.e., zero discrimination between familiar and novel objects).

impaired on AP-5 trials relative to saline trials with the $180 \mathrm{~min}$ retention delay $\left(t_{(10)}=6.27 ; p<0.001\right)$ but not with the $5 \mathrm{~min}$ delay $(t<1)$. These results indicate that NMDAR activation during the sample phase is required for subsequent object recognition memory if the retention delay is relatively long. NMDAR activity is not necessary, however, for encoding or retention of object information over short intervals.

\section{Experiment 2: AMPAR blockade during encoding}

To assess the effects of AMPAR blockade during encoding, we infused saline or the AMPAR antagonist CNQX into PRh 15 min before the sample phase and tested rats with a $5 \mathrm{~min}$ and a 180 min retention delay as in experiment 1 . The drug did not affect measures of general object exploration in the sample or choice phases but significantly disrupted object recognition with both retention delays (Fig. 4). Analysis of the total time required to sample the objects for $25 \mathrm{~s}$ revealed no significant effects of drug $\left(F_{(1,10)}=1.07 ; p>0.10\right)$, delay $\left(F_{(1,10)}=1.28 ; p>0.10\right)$, or the interaction $\left(F_{(1,10)}=2.24 ; p>0.10\right)$ (means \pm SEM: saline, 5 $\min =135.62 \pm 12.55 \mathrm{~s}$; saline, $180 \mathrm{~min}=101.64 \pm 14.70 \mathrm{~s}$; $\mathrm{CNQX}, 5 \mathrm{~min}=126.30 \pm 12.43 \mathrm{~s}$; CNQX, $180 \mathrm{~min}=142.85 \pm$ $21.61 \mathrm{~s})$. Moreover, there was no effect of drug $(F<1)$ or delay $\left(F_{(1,10)}=3.2 ; p>0.10\right)$ on the total amount of time spent by rats exploring the objects in the choice phase, nor was the drug by delay interaction significant $\left(F_{(1,10)}=4.26 ; p>0.05\right)$ (means \pm SEM: saline, $5 \mathrm{~min}=12.44 \pm 1.64 \mathrm{~s}$; saline, $180 \mathrm{~min}=13.69 \pm$ $1.17 \mathrm{~s}$; CNQX, $5 \mathrm{~min}=15.87 \pm 2.69 \mathrm{~s}$; CNQX, $180 \mathrm{~min}=9.74 \pm$ $1.12 \mathrm{~s})$. Intra-PRh infusions of CNQX, however, severely disrupted object recognition memory regardless of the length of the retention delay. Analysis of the discrimination ratio revealed significant effects of drug $\left(F_{(1,10)}=19.97 ; p<0.01\right)$ and the drug by delay interaction $\left(F_{(1,10)}=5.77 ; p<0.05\right)$; the delay effect was not significant $(F<1)$. Post hoc comparisons of the two drug conditions at each delay indicated that performance under CNQX was significantly disrupted at both the $5 \mathrm{~min}\left(t_{(10)}=4.82\right.$; $p<0.01)$ and $180 \min \left(t_{(10)}=2.67 ; p<0.05\right)$ retention delays. 


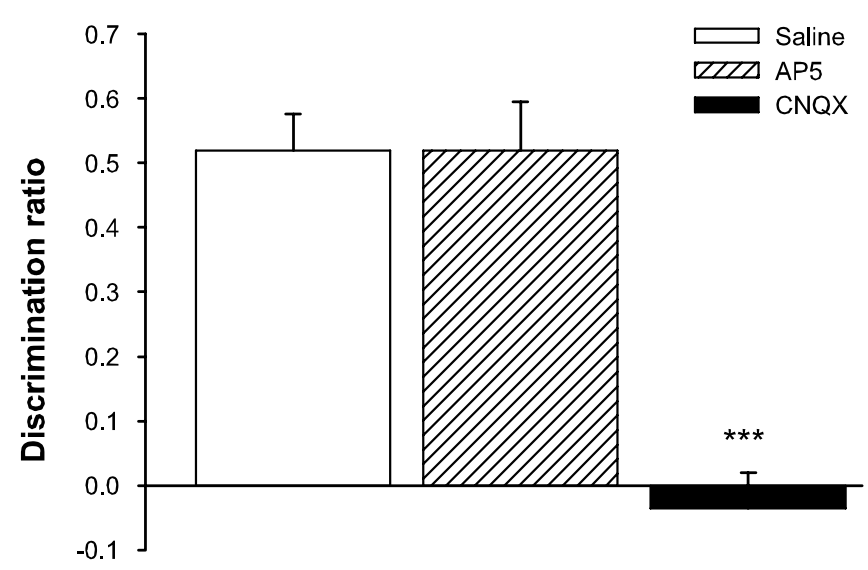

Figure 5. AMPAR but not NMDAR blockade in PRh disrupts retrieval in object recognition memory. Spontaneous object recognition performance by animals in experiment 3 on trials in which they received intra-PRh infusions of AP-5, CNQX, or saline 15 min before the choice phase. The retention delay was 180 min. Rats were impaired on CNQX trials, but AP-5 had no effect. Data are presented as average discrimination ratio \pm SEM. ${ }^{* * *} p<0.001$, CNQX versus saline and CNQX versus AP-5.

These results suggest that AMPAR activity during the sampling phase is required for both short- and long-term object recognition memory.

\section{Experiment 3: NMDAR and AMPAR blockade during retrieval}

Experiments 1 and 2 indicate dissociable roles for AMPARs and NMDARs in object recognition memory. Whereas AMPAR activity during the sample phase is required for subsequent shortand long-term object recognition memory, NMDAR activity is necessary only when the information acquired during the sample phase must be retained for a relatively long time. This finding is consistent with the established role for NMDARs in the induction of activity-dependent synaptic plasticity processes that may mediate long-term memory (Bliss and Collingridge, 1993). AMPARs are important for fast synaptic transmission and may, therefore, play a more general role in information processing within PRh. Thus, the effects observed in experiment 2 may indicate involvement of AMPARs in perceptual aspects of object encoding. To examine further the roles of these receptor types in object recognition memory, we next compared the effects of intra-PRh AP-5 and CNQX when the infusions were performed $15 \mathrm{~min}$ before the choice phase after a retention delay of $180 \mathrm{~min}$. This allowed us to compare the effects of NMDA and AMPA receptor blockade at the time of retrieval.

Drug infusions had no effects on the time taken to explore the objects in the sample phase $(F<1$; means \pm SEM: saline $=$ $82.67 \pm 4.73 \mathrm{~s} ; \mathrm{AP}-5=89.03 \pm 8.39 \mathrm{~s} ; \mathrm{CNQX}=79.91 \pm 5.94 \mathrm{~s})$ or the total exploration time in the choice phase $(F<1$; means \pm SEM: saline $=15.85 \pm 1.07 \mathrm{~s} ; \mathrm{AP}-5=13.92 \pm 1.53 \mathrm{~s}$; CNQX $=$ $14.70 \pm 1.02 \mathrm{~s}$ ). Intra-PRh infusions of CNQX, however, severely disrupted object recognition memory when compared with performance on saline and AP-5 trials (Fig. 5). Analysis of the discrimination ratio yielded a significant drug effect $\left(F_{(2,22)}=24.11\right.$; $p<0.001)$, and post hoc comparisons indicated that performance under CNQX differed significantly from saline $\left(t_{(11)}=6.2 ; p<\right.$ $0.001)$ and $\mathrm{AP}-5\left(t_{(11)}=7.27 ; p<0.001\right)$ trials, whereas object recognition on AP-5 trials was similar to that under saline $(t<1)$. These results suggest additional functional dissociations between AMPARs and NMDARs in object recognition memory. AMPAR,

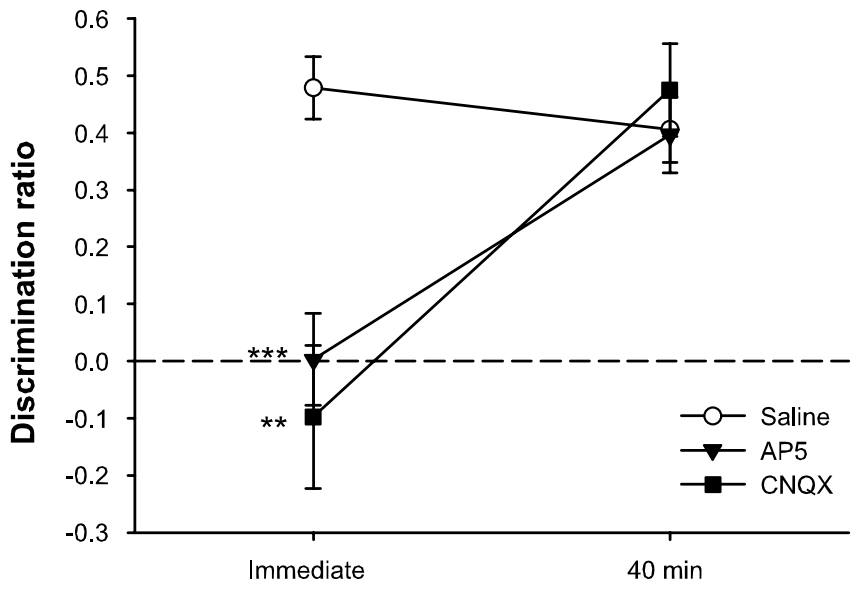

Post-sample infusion time

Figure 6. AMPAR or NMDAR blockade in PRh disrupts consolidation of object memory. Spontaneous object recognition performance by animals in experiment 4 on trials in which they received intra-PRh infusions of AP-5, CNQX, or saline immediately or 40 min following the sample phase. The retention delay was $180 \mathrm{~min}$. Both AP-5 and CNQX impaired object recognition when infused immediately after the sample phase but not when the infusions were delayed by $40 \mathrm{~min}$. Data are presented as average discrimination ratio \pm SEM. ${ }^{* *} p<0.01$, CNQX versus saline; ${ }^{* * *} p<0.001, A P-5$ versus saline. The dashed line indicates chance performance (i.e., zero discrimination between familiar and novel objects).

but not NMDAR, activity is necessary within PRh at the time of retrieval.

\section{Experiment 4: NMDAR and AMPAR blockade during consolidation}

We found previously that inactivation of PRh with lidocaine disrupts subsequent object recognition when infusions are made immediately or $20 \mathrm{~min}$ after the end of the sample phase; intraPRh lidocaine has no effect, however, if infusions are delayed until 40 min postsample (Winters and Bussey, 2005). This pattern of results suggests a consolidation period during which the memory trace becomes stabilized within PRh after the sample phase. In the present experiment, we wished to determine whether AMPA and/or NMDA receptors play a role in this sensitive phase. The goal of this experiment was specifically to determine whether these glutamate receptors play a role in the earliest stages of storage or consolidation taking place immediately after the removal of the sample stimulus and, if so, whether this contribution follows a similar time course to that demonstrated for general PRh activity with lidocaine. Thus, we infused AP-5, CNQX, or saline into PRh immediately or 40 min after the sample phase and tested object recognition $180 \mathrm{~min}$ after the sample phase. Previous electrophysiological findings suggest that this retention delay should allow sufficient time for the effects of CNQX on synaptic transmission to dissipate before the start of the choice phase (Day et al., 2003).

Although the drugs did not differentially affect measures of general object exploration, immediate, but not $40 \mathrm{~min}$, postsample infusions of either AP-5 or CNQX significantly disrupted object recognition performance (Fig. 6). Analysis of the total time required to explore the sample objects for $25 \mathrm{~s}$ revealed no significant effects of drug $\left(F_{(2,22)}=2.39 ; p>0.10\right)$, but the effect of infusion time was significant $\left(F_{(1,11)}=14.08\right.$; $\left.p<0.01\right)$. Importantly, however, the drug by infusion time interaction was not 
significant $\left(F_{(2,22)}=1.33 ; p>0.10\right)$ (means \pm SEM: saline, immediate $=74.98 \pm 9.06 \mathrm{~s}$; saline, $40 \mathrm{~min}=117.28 \pm 9.25 \mathrm{~s}$; AP-5, immediate $=65.81 \pm 5.79 \mathrm{~s} ; \mathrm{AP}-5,40 \mathrm{~min}=91.13 \pm 12.93 \mathrm{~s}$; CNQX, immediate $=72.30 \pm 7.41 \mathrm{~s}$; CNQX, $40 \mathrm{~min}=88.72 \pm$ $10.05 \mathrm{~s})$. There was also a significant effect of infusion time on total choice exploration $\left(F_{(1,11)}=8.22 ; p<0.05\right)$, but neither the drug effect nor the drug by infusion time interaction was significant (both $F<1$; means \pm SEM: saline, immediate $=15.14 \pm$ $1.28 \mathrm{~s}$; saline, $40 \mathrm{~min}=14.15 \pm 1.24 \mathrm{~s}$; AP-5, immediate $=$ $15.55 \pm 1.78 \mathrm{~s}$; AP-5, $40 \mathrm{~min}=11.66 \pm 1.33 \mathrm{~s}$; CNQX, immediate $=14.95 \pm 1.33 \mathrm{~s}$; CNQX, $40 \mathrm{~min}=12.27 \pm 1.26 \mathrm{~s}$ ). Analysis of the discrimination ratio, however, revealed significant effects of drug $\left(F_{(2,22)}=6.93 ; p<0.01\right)$, infusion time $\left(F_{(1,11)}=14.45\right.$; $p<0.01)$, and a significant interaction $\left(F_{(2,22)}=11.04 ; p<\right.$ 0.001). Post hoc analyses indicated that object recognition was significantly disrupted by both AP-5 $\left(t_{(11)}=5.31 ; p<0.001\right)$ and CNQX $\left(t_{(11)}=4.15 ; p<0.01\right)$ in the immediate post-sample infusion condition when compared with performance on saline trials. In the $40 \mathrm{~min}$ post-sample infusion condition, rats performed as well on AP-5 and CNQX trials as they did on saline trials (both $t<1$ ). These results indicate that AMPAR and NMDAR activity is necessary for a limited period after the end of the sample phase for normal subsequent object recognition memory.

\section{Discussion}

The present study provides additional evidence for the involvement of PRh in three distinct stages of object recognition memory: encoding, retrieval, and consolidation. Moreover, these results indicate different roles for AMPA and NMDA glutamate receptors in PRh in these three stages of memory processing. This pattern of results is the first to demonstrate such functional dissociations within $\mathrm{PRh}$ and, as such, provides useful insight into our understanding of this important memory processing area and the roles of AMPARs and NMDARs in learning and memory.

\section{Perirhinal AMPARs and NMDARs in object encoding}

Infusions of CNQX into PRh before the sample phase significantly impaired object recognition memory compared with the same animals' performance on trials in which they received saline before the sample phase. This effect was seen with relatively short (5 $\mathrm{min}$ ) and long (180 $\mathrm{min}$ ) retention delays. These results suggest a significant role for PRh AMPARs in the initial encoding of the object trace and are consistent with previous reports of impaired memory acquisition caused by pretraining administration of AMPAR antagonists in other behavioral tasks. For example, Riedel et al. (1999) reported that intrahippocampal infusions of the AMPA/kainite receptor antagonist LY326325 (a decahydroisoquiniline compound) disrupted acquisition of spatial memory in the water maze, and Day et al. (2003) showed that intrahippocampal CNQX impaired encoding in a flavor-place paired-associates task. Moreover, Izquierdo et al. (1998b) showed that the acquisition of step-down inhibitory avoidance learning can be disrupted by pretraining infusions of CNQX into the hippocampus. These results, with the present findings, indicate a role for AMPARs in different brain regions in encoding and acquisition of different types of information.

We cannot entirely rule out the possibility that some postsample AMPAR blockade may have contributed to the deficits observed in pre-sample CNQX conditions, because post-sample CNQX infusions impaired object recognition memory in experiment 4. Electrophysiological findings demonstrate that the maximal level of neural inactivation induced by CNQX in the hip- pocampus in vitro occurs 10-15 min after application of the drug (Day et al., 2003). For this reason, we chose a $15 \mathrm{~min}$ pre-sample infusion time. However, Day et al. (2003) also reported that the neural inactivation caused by CNQX can last for $\sim 60$ min before neural transmission returns to baseline. It thus remains a possibility that pre-sample infusions of CNQX also disrupted PRh transmission for a time after the sample phase. Additional experiments combining intra-PRh CNQX infusions with electrophysiology may be required to resolve this issue.

The suggestion that AMPAR-mediated synaptic transmission in PRh is necessary for encoding is consistent with many recent studies implicating PRh in object identification and perceptual representation (Buckley and Gaffan, 1998a,b; Murray and Bussey, 1999; Buckley et al., 2001; Bussey and Saksida, 2002; Bussey et al., 2002a). Indeed, it is well established that PRh lesions can disrupt visual discrimination as well as object recognition (Buckley and Gaffan, 1997; Buckley et al., 2001; Eacott et al., 2001; Bussey et al., 2002b, 2003). Such findings suggest that PRh stores complex representations of objects (Buckley and Gaffan, 1998b; Bussey and Saksida, 2002). The effects of pre-sample blockade of PRh AMPARs may, therefore, indicate interference with encoding of the perceptual representation of the object (Dudai, 2002).

In contrast, blockade of NMDARs with AP-5 in PRh during the sample phase disrupted only long-term object recognition memory (180 min retention delay), leaving intact performance when the delay was relatively short ( $5 \mathrm{~min}$ ). This result suggests that PRh NMDAR activity is not necessary for the initial perceptual encoding of the object trace. Rather, NMDAR activity within $\mathrm{PRh}$ at the time of encoding is probably required for the induction of activity-dependent synaptic plasticity processes important for long-term storage (Martin et al., 2000). The present results suggest that previously reported object recognition deficits after systemic pre-sample injections of the NMDAR antagonist (+)-5-methyl-10,11-dihydro-5H-dibenzo [a,d] cyclohepten5,10-imine maleate (MK-801) with relatively long retention delays (de Lima et al., 2005) may have been caused by blockade of NMDARs in PRh. Moreover, the present findings are consistent with previous studies reporting delay-dependent effects of pretraining intrahippocampal AP-5 infusions in spatial and contextual learning and memory tasks (Kim et al., 1992; Steele and Morris, 1999). The present findings indicate that, as in the hippocampus for spatial memory, NMDARs in PRh are crucially involved in the neural mechanisms required for long-term object memory.

Perirhinal AMPARs and NMDARs in object memory retrieval Although blockade of PRh NMDARs at the time of encoding disrupted object recognition 180 min later, NMDAR activity was not necessary in PRh at the time of retrieval after this longer retention delay, consistent with a selective role in activitydependent synaptic plasticity. In contrast, the AMPAR antagonist CNQX severely disrupted object recognition when infused into PRh before the choice phase, consistent with previous findings with different behavioral tasks after pretest AMPAR blockade in other brain regions (Izquierdo et al., 1997; Riedel et al., 1999; Day et al., 2003). The differential pattern of results seen with pre-sample versus pre-choice intra-PRh AP-5 infusions mirrors that seen after intrahippocampal infusions in other tasks. For example, Day et al. (2003) reported that, although intrahippocampal AP-5 impaired encoding, it did not disrupt retrieval in the paired-associates task, whereas CNQX affected both stages. Kim et al. (1991) have also shown, in a contextual fear conditioning paradigm, that systemic AP-5 blocks memory acquisition but 
not expression. The present findings further illustrate the functional differences between PRh AMPARs and NMDARs and provide additional support for the involvement of PRh synaptic transmission at the time of object memory retrieval.

\section{Perirhinal AMPARs and NMDARs in object memory consolidation}

Blockade of either AMPARs or NMDARs in PRh immediately but not 40 min after the sample phase abolished subsequent object recognition memory. These results suggest, consistent with our previous findings with post-sample intra-PRh lidocaine infusions (Winters and Bussey, 2005), that the object trace encoded during the sample phase is dependent on activity within PRh for a limited period of time after the end of the sample phase. Similarly, previous studies have reported memory deficits in different behavioral tasks after immediate post-training infusions of AMPAR or NMDAR antagonists into other areas of the brain (Izquierdo et al., 1997, 1998a,b; Riedel et al., 1999), and de Lima et al. (2005) showed recently that post-sample systemic injections of the NMDAR antagonist MK-801 can disrupt spontaneous object recognition with retention delays of 1.5 or $24 \mathrm{~h}$. The present findings indicate that both AMPAR and NMDAR activity is necessary for successful consolidation of object memory within PRh. AMPAR-mediated synaptic transmission may be required to maintain the encoded trace, whereas NMDAR activity contributes to the establishment of synaptic changes required for longerterm storage (Goelet et al., 1986; Dudai, 1996; Martin et al., 2000). This consolidation period seems to end within the first 40 min after encoding.

As discussed previously, teasing apart the effects of pre-sample and post-sample infusions in the present study is complicated by the fact that pre-sample infusions likely lead to some post-sample receptor antagonism as a result of the time course of the drugs. The maximal effects of post-sample drugs were unlikely, however, to have occurred until 15 or $20 \mathrm{~min}$ after the immediate post-sample infusion, whereas the maximal effects of pre-sample drugs would be attained at the outset of the sample phase. Furthermore, and importantly, the post-sample infusion effects of CNQX and AP-5 demonstrate that AMPAR and NMDAR activity is still required after the stimulus is no longer physically present. The CNQX- and AP-5-induced impairments, thus, indicate that the functions of AMPARs and NMDARs in PRh are not required just for the superficial encoding that takes place during the sample phase but also for the processing taking place after removal of the stimulus. Although this processing may be linked to continued firing of PRh neurons after the sample phase, this is in the absence of the sample stimulus and can, thus, be thought of as an aspect of memory storage and early consolidation. Whether this glutamate receptor dependence continues into the delay, for example at a 20 min post-sample infusion time, as we have shown for the effects of lidocaine (Winters and Bussey, 2005) cannot be determined from the present findings. Such an analysis would, however, be of interest, because the results could add further weight to the evidence presented here for involvement of AMPARs and NMDARs in object memory storage and consolidation.

Long-term potentiation (LTP) and long-term depression (LTD) are two forms of activity-dependent synaptic plasticity that involve AMPARs and NMDARs (Bliss and Collingridge, 1993; Ziakopoulos et al., 1999; Cho et al., 2000; Martin et al., 2000). Moreover, both NMDAR-dependent LTP and LTD occur in PRh (Bilkey, 1996; Ziakopoulos et al., 1999), and these provide likely candidates for at least part of the neural mechanism re- quired for object recognition memory (Brown and Bashir, 2002). It is possible that the mechanisms of AMPARs and NMDARs interact with metabotropic receptors to mediate long-term retention of object memory, because it has been shown that a form of activity-dependent LTD within PRh relies on interaction between NMDARs and metabotropic glutamate receptors (Cho et al., 2000). The present results indicate that initial encoding of the object trace in PRh does not ensure the completion of cellular and molecular changes required for long-term storage. Rather, the trace gradually transfers from a labile state, which may require ongoing AMPAR-mediated synaptic transmission, to a more resistant memory, the consolidation of which requires NMDAR activity after the sample phase.

Although we have not performed an analysis of the actual spread of drug effects in the present study, we can be quite certain that this was limited to PRh. Using similar infusion parameters with radiolabeled lidocaine, we have shown that infusate spreads rostrocaudally along the full length of PRh without encroaching substantially into other temporal lobe regions (Winters and Bussey, 2005). Nonetheless, there is a small chance that the important negative results with AP-5 in the present study were attributable to partial NMDAR blockade within the elongated PRh. Because we have not analyzed specifically AP-5 or CNQX spread, we cannot be absolutely certain that receptor blockade within PRh was complete with these drugs. However, the positive results from the same experiments using identical infusion parameters provide control conditions that argue against an explanation of the negative results based on partial receptor blockade within PRh.

\section{Conclusion}

Object recognition tests are increasingly prevalent in basic and preclinical research studies of the mechanisms of memory. Here, we show that this important mnemonic process comprises interdependent but distinct stages, all of which depend on neural transmission within PRh. The pattern of spared and impaired performance in this study contributes importantly to our understanding of PRh function. Specifically, the use of CNQX provides an important extension of previous results with lidocaine (Winters and Bussey, 2005). The specificity of CNQX allows us to conclude more confidently that cellular activity within PRh is involved in the distinct stages of object recognition memory. Indeed, the advantage provided by the present results over those with lidocaine is akin to the difference between excitotoxic versus radiofrequency or aspiration lesions.

In addition to confirming the role of PRh in the stages of object recognition, the present results emphasize the importance of glutamate receptors and their different contributions to these processes. The present findings suggest a crucial role for AMPAR-mediated synaptic transmission within PRh during the encoding, retrieval, and consolidation of object memory. In contrast, NMDAR activity is not necessary at the retrieval stage or for encoding short-term memory. Rather, NMDAR activity within PRh seems to be important for the induction of processes during encoding and early consolidation that are necessary for longterm retention of the object memory trace. The pattern of the present results is consistent with findings in other brain areas and different behavioral tasks (Miserendino et al., 1990; Kim et al., 1991, 1992, 1993; Day et al., 2003), suggesting a degree of generality in the mechanisms of learning and memory.

\section{References}

Bartolini L, Casamenti F, Pepeu G (1996) Aniracetam restores object recognition impaired by age, scopolamine, and nucleus basalis lesions. Pharmacol Biochem Behav 53:277-283. 
Bilkey DK (1996) Long-term potentiation in the in vitro perirhinal cortex displays associative properties. Brain Res 733:297-300.

Bliss TV, Collingridge GL (1993) A synaptic model of memory: long-term potentiation in the hippocampus. Nature 361:31-39.

Bliss TVP, Lomo T (1973) Long-lasting potentiation of synaptic transmission in the dentate area of the anaesthetized rabbit following stimulation of the performant path. J Physiol (Lond) 232:331-356.

Bozon B, Kelly A, Josselyn SA, Silva AJ, Davis S, Laroche S (2003) MAPK, CREB and zif268 are all required for the consolidation of recognition memory. Philos Trans R Soc Lond B Biol Sci 358:805-814.

Brown MW, Bashir ZI (2002) Evidence concerning how neurons of the perirhinal cortex may effect familiarity discrimination. Philos Trans R Soc Lond B Biol Sci 357:1083-1095.

Buckley MJ, Gaffan D (1997) Impairment of visual object-discrimination learning after perirhinal cortex ablation. Behav Neurosci 111:467-475.

Buckley MJ, Gaffan D (1998a) Learning and transfer of object-reward associations and the role of the perirhinal cortex. Behav Neurosci 112:15-23.

Buckley MJ, Gaffan D (1998b) Perirhinal cortex ablation impairs visual object identification. J Neurosci 18:2268-2275.

Buckley MJ, Booth MC, Rolls ET, Gaffan D (2001) Selective perceptual impairments after perirhinal cortex ablation. J Neurosci 21:9824-9836.

Buffalo EA, Reber PJ, Squire LR (1998) The human perirhinal cortex and recognition memory. Hippocampus 8:330-339.

Burwell RD (2001) Borders and cytoarchitecture of the perirhinal and postrhinal cortices in the rat. J Comp Neurol 437:17-41.

Bussey TJ, Saksida LM (2002) The organization of visual object representations: a connectionist model of effects of lesions in perirhinal cortex. Eur J Neurosci 15:355-364.

Bussey TJ, Muir JL, Aggleton JP (1999) Functionally dissociating aspects of event memory: the effects of combined perirhinal and postrhinal cortex lesions on object and place memory in the rat. J Neurosci 19:495-502.

Bussey TJ, Duck J, Muir JL, Aggleton JP (2000) Distinct patterns of behavioural impairments resulting from fornix transection or neurotoxic lesions of the perirhinal and postrhinal cortices in the rat. Behav Brain Res 111:187-202.

Bussey TJ, Saksida LM, Murray EA (2002a) The role of perirhinal cortex in memory and perception: conjunctive representations for object identification. In: The parahippocampal region: organization and role in cognitive functions (Witter MP, Wouterlood FG, eds), pp 239-254. Oxford: Oxford UP.

Bussey TJ, Saksida LM, Murray EA (2002b) Perirhinal cortex resolves feature ambiguity in complex visual discriminations. Eur J Neurosci 15:365-374.

Bussey TJ, Saksida LM, Murray EA (2003) Impairments in visual discrimination after perirhinal cortex lesions: testing "declarative" vs. "perceptual-mnemonic" views of perirhinal cortex function. Eur J Neurosci 17:649-660.

Cho K, Kemp N, Noel J, Aggleton JP, Brown MW, Bashir ZI (2000) A new form of long-term depression in the perirhinal cortex. Nat Neurosci 3:150-156.

Day M, Langston R, Morris RG (2003) Glutamate-receptor-mediated encoding and retrieval of paired-associate learning. Nature 424:205-209.

de Lima MN, Laranja DC, Bromberg E, Roesler R, Schroder N (2005) Preor post-training administration of the NMDA receptor blocker MK-801 impairs object recognition memory in rats. Behav Brain Res 156:139-143.

Dudai Y (1996) Consolidation: fragility on the road to the engram. Neuron $17: 367-370$

Dudai Y (2002) Memory from A to Z: keywords, concepts, and beyond, pp 6-8. Oxford: Oxford UP.

Duzel E, Habib R, Rotte M, Guderian S, Tulving E, Heinze HJ (2003) Human hippocampal and parahippocampal activity during visual associative recognition memory for spatial and nonspatial stimulus configurations. J Neurosci 23:9439-9444.

Eacott MJ, Machin PE, Gaffan EA (2001) Elemental and configural visual discrimination learning following lesions to perirhinal cortex in the rat. Behav Brain Res 124:55-70.

Elliott R, Dolan RJ (1998) The neural response in short-term visual recognition memory for perceptual conjunctions. NeuroImage 7:14-22.
Ennaceur A, Delacour J (1988) A new one-trial test for neurobiological studies of memory in rats. 1: Behavioral data. Behav Brain Res 31:47-59.

Ennaceur A, Neave N, Aggleton JP (1996) Neurotoxic lesions of the perirhinal cortex do not mimic the behavioural effects of fornix transection in the rat. Behav Brain Res 80:9-25.

Forwood SE, Winters BD, Bussey TJ (2005) Hippocampal lesions that abolish spatial maze performance spare object recognition memory at delays of up to 48 hours. Hippocampus 15:347-355.

Gaffan D (1994) Dissociated effects of perirhinal cortex ablation, fornix transection and amygdalectomy: evidence for multiple memory systems in the primate temporal lobe. Exp Brain Res 99:411-422.

Goelet P, Castellucci VF, Schacher S, Kandel ER (1986) The long and the short of long-term memory-a molecular framework. Nature 322:419-422.

Izquierdo I, Quillfeldt JA, Zanatta MS, Quevedo J, Schaeffer E, Schmitz PK, Medina JH (1997) Sequential role of hippocampus and amygdala, entorhinal cortex and parietal cortex in formation and retrieval of memory for inhibitory avoidance in rats. Eur J Neurosci 9:786-793.

Izquierdo I, Barros DM, Mello e Souza T, de Souza MM, Izquierdo LA, Medina JH (1998a) Mechanisms for memory types differ. Nature 393:635-636.

Izquierdo I, Izquierdo LA, Barros DM, Mello e Souza T, de Souza MM, Quevedo J, Rodrigues C, Sant'Anna MK, Madruga M, Medina JH (1998b) Differential involvement of cortical receptor mechanisms in working, short-term and long-term memory. Behav Pharmacol 9:421-427.

Kim JJ, DeCola JP, Landeira-Fernandez J, Fanselow MS (1991) N-methylD-aspartate receptor antagonist APV blocks acquisition but not expression of fear conditioning. Behav Neurosci 105:126-133.

Kim JJ, Fanselow MS, DeCola JP, Landeira-Fernandez J (1992) Selective impairment of long-term but not short-term conditional fear by the $\mathrm{N}$-methyl-D-aspartate antagonist APV. Behav Neurosci 106:591-596.

Kim M, Campeau S, Falls WA, Davis M (1993) Infusion of the non-NMDA receptor antagonist CNQX into the amygdala blocks the expression of fear-potentiated startle. Behav Neural Biol 59:5-8.

Martin SJ, Grimwood PD, Morris RGM (2000) Synaptic plasticity and memory: an evaluation of the hypothesis. Annu Rev Neurosci 23:649-711.

McKee RD, Squire LR (1993) On the development of declarative memory. J Exp Psychol Learn Mem Cogn 19:397-404.

Meunier M, Bachevalier J, Mishkin M, Murray EA (1993) Effects on visual recognition of combined and separate ablations of the entorhinal and perirhinal cortex in rhesus monkeys. J Neurosci 13:5418-5432.

Miserendino MJ, Sananes CB, Melia KR, Davis M (1990) Blocking of acquisition but not expression of conditioned fear-potentiated startle by NMDA antagonists in the amygdala. Nature 345:716-718.

Mishkin M (1978) Memory in monkeys severely impaired by combined but not by separate removal of amygdala and hippocampus. Nature 273:297-298.

Mumby DG, Pinel JP (1994) Rhinal cortex lesions and object recognition in rats. Behav Neurosci 108:11-18.

Murray EA, Bussey TJ (1999) Perceptual-mnemonic functions of the perirhinal cortex. Trends Cogn Sci 3:142-151.

Murray EA, Mishkin M (1998) Object recognition and location memory in monkeys with excitotoxic lesions of the amygdala and hippocampus. J Neurosci 18:6568-6582.

Nemanic S, Alvarado MC, Bachevalier J (2004) The hippocampal/parahippocampal regions and recognition memory: insights from visual paired comparison versus object-delayed nonmatching in monkeys. J Neurosci 24:2013-2026.

Pascalis O, Bachevalier J (1999) Neonatal aspiration lesions of the hippocampal formation impair visual recognition memory when assessed by paired-comparison task but not by delayed nonmatching-to-sample task. Hippocampus 9:609-616.

Paxinos G, Watson C (1997) The rat brain in stereotaxic coordinates, Ed 3. London: Academic.

Riedel G, Micheau J, Lam AG, Roloff E, Martin SJ, Bridge H, Hoz L, Poeschel B, McCulloch J, Morris RGM (1999) Reversible neural inactivation reveals hippocampal participation in several memory processes. Nat Neurosci 2:898-905.

Steele RJ, Morris RGM (1999) Delay-dependent impairment of a matching- 
to-place task with chronic and intrahippocampal infusion of the NMDAantagonist D-AP5. Hippocampus 9:118-136.

Tang YP, Shimizu E, Dube GR, Rampon C, Kerchner GA, Zhuo M, Liu G, Tsien JZ (1999) Genetic enhancement of learning and memory in mice. Nature 401:63-69.

Winters BD, Bussey TJ (2005) Transient inactivation of perirhinal cortex disrupts encoding, retrieval, and consolidation of object recognition memory. J Neurosci 25:52-61.

Winters BD, Forwood SE, Cowell RA, Saksida LM, Bussey TJ (2004) Double dissociation between the effects of peri-postrhinal cortex and hippocampal lesions on tests of object recognition and spatial memory: heterogeneity of function within the temporal lobe. J Neurosci 24:5901-5908.

Ziakopoulos Z, Tillett CW, Brown MW, Bashir ZI (1999) Input- and layer- dependent synaptic plasticity in the rat perirhinal cortex in vitro. Neuroscience 92:459-472.

Zola-Morgan S, Squire LR (1985) Medial temporal lesions in monkeys impair memory on a variety of tasks sensitive to human amnesia. Behav Neurosci 99:22-34.

Zola-Morgan S, Squire LR, Amaral DG (1986) Human amnesia and the medial temporal region: enduring memory impairment following a bilateral lesion limited to field CA1 of the hippocampus. J Neurosci 6:2950-2967.

Zola-Morgan S, Squire LR, Amaral DG, Suzuki WA (1989) Lesions of perirhinal and parahippocampal cortex that spare the amygdala and hippocampal formation produce severe memory impairment. J Neurosci 9:4355-4370. 